For personal use only. Not to be reproduced without permission of the publisher (editorial@gabi-journal.net).

\section{International Alliance of Patients' Organizations toolkit for biological and biosimilar medicines}

The International Alliance of Patients' Organizations (IAPO) is an alliance of over 200 patient groups that represents the interests of patients worldwide. In November 2013, IAPO published its Information and Advocacy Toolkit on Biological and Biosimilar Medicines for Patients' Organizations [1]. The Toolkit was developed to provide up-to-date, evidence-based information on the science, technology, and regulatory information relevant to biological and biosimilar medicines.

IAPO believes patients should be aware of what biological and biosimilar medicines are and the implications of their increasing availability. The Toolkit was developed to allow patient advocates to make informed judgements on the value of biological and biosimilar medicines and actively engage in debate and discussion with other stakeholders involved in health care.

The Toolkit contains a briefing paper on biological and biosimilar medicines, a quick guide, a guide on what patients' organizations can do, as well as a number of fact sheets. It is available free of charge to patients' organizations around the world. It is unique in that it explores the perspectives of a variety of different stakeholders including patients' organizations, regulators, medical associations, academics and biological and biosimilar medicine manufacturers.

IAPO's Toolkit covers important issues for patients' organizations and other stakeholders, identified through an online consultation and through in-depth interviews. Topics include the safety of biological medicines, how they are regulated and monitored, how they are prescribed and dispensed, as well as who can access them and what information and support is available to patients.

In December 2012, IAPO held an online consultation with its members to understand the level of awareness of biological and biosimilar medicines, their regulation and use, and the issues and concerns that patients' organizations found most important. Raising patient awareness is essential, since the acceptance of generics substitution worldwide has been slow, with research showing that many patients get confused or feel apprehensive about having their drugs changed [2].

IAPO found varied awareness of biosimilar medicines among respondents. Most (54\%) of the patients' organizations that responded represented patients who were currently using biological medicines for treatment of their disease, and $20 \%$ of the respondents represented patients who were using biosimilar medicines. The majority of respondents had no (36\%) or some (32\%) awareness of how biosimilar medicines were regulated in their country or region.

Ensuring the safety of biosimilar medicines is an important issue for patients' organizations. Safety includes a broad range of issues from how biosimilars are defined and named, to their ability to cause immune reactions, regulation and pharmacovigilance.

Pharmacovigilance is critical in ensuring the safety of all medicines. As all biological medicines can potentially cause an immune response after approval, surveillance and monitoring are absolutely essential to track any adverse effects caused by the medicine [3, 4].

IAPO's online consultation revealed that $50 \%$ of respondents had some to high awareness of the pharmacovigilance system in their country while 33\% had little awareness and 19\% had no awareness at all. A number of patients' organizations from both developed and developing countries highlighted the importance of a strong pharmacovigilance system, and how this was lacking in their countries.

Pharmacovigilance depends on being able to track and trace biological and biosimilar medicines. Being able to differentiate between medicines is essential [5]. Manufacturers of medicines can apply to the World Health Organization for an International Nonproprietary Name (INN). INNs facilitate the identification of pharmaceutical substances or active ingredients in medicines [6]. Each unique INN, sometimes called a generic name, is different to the brand name but shared between identical pharmaceutical substances. INNs are important for the clear identification, safe prescription and dispensing of medicines to patients, and for communication and exchange of information among healthcare professionals and scientists. However, the structural complexity of biological medicines means that a biosimilar cannot be identical to the original biological medicine or to other biosimilars.

If doctors or pharmacists use only the INN when prescribing a biological or biosimilar medicine, and if several biosimilars exist, it will not be known exactly which medicine the patient is being given. If an adverse effect occurs it will not be clear which medicine - either the original biological or any of several biosimilars - caused it [4]. Automatic substitution of approved biosimilars by the pharmacist without notifying the patient and physician/healthcare provider would circumvent pharmacovigilance, putting patient safety at risk [7], as could prescribing medicines using only the brand name.

Each biosimilar medicine should have a unique identifier (or brand name) in addition to its INN to make it clear which medicine a patient is taking $[8,9]$. Patients should ensure that when they are prescribed a biological or biosimilar medicines they know the unique identifier (or brand name), manufacturer's name and where to find the batch number of their medicine.

Biological medicines have revolutionized the treatment of many diseases and have benefited millions of patients worldwide. IAPO believes that ensuring access to high quality, safe and efficacious biological and biosimilar medicines depends on education of patients, doctors and health authorities. Stringent regulatory guidelines based on those of the European Medicines Agency, World Health Organization or US Food and Drug Administration, and robust pharmacovigilance and adverse event monitoring systems are key to ensuring patient safety.

To see the Toolkit and for more information on IAPO's work on biological and biosimilar medicines please visit www.patientsorganizations.org/biosimilars

\section{Competing interests: None.}


PHARMA NEWS

Provenance and governance: Article abstracted based on the summary paper prepared by Ms Yasemin Dil, IAPO, UK; internally peer reviewed.

Bea Perks, PhD, GaBI Journal Editor

\section{References}

1. International Alliance of Patients' Organizations (IAPO). Biological and biosimilar medicines: an information and advocacy toolkit for patients' organizations, 2013 [homepage on the Internet]. [cited 2014 Sep 9]. Available from: http:// www.patientsorganizations.org $/$ showarticle.pl?id=1763\&n=961

2. Håkonsen H, Toverud E-L. A review of patient perspectives on generics substitution: what are the challenges for optimal drug use. Generics and Biosimilars Initiative Journal (GaBI Journal). 2012;1(1):28-32. doi:10.5639/gabij.2012. 0101.008

3. EuropaBio. Guide to biosimilars: a focus on biosimilar medicines. 26 October 2011 [homepage on the Internet]. [cited 2014 Sep 9]. Available from: http:// www.europabio.org/guide-biological-medicines-focus-biosimilar-medicines

4. Schellekens H. How similar do 'biosimilars' need to be? Nat Biotechnol. 2004; 22(11): 1357-9.

5. Mellstedt H, Niederwieser D, Ludwig, H. The challenge of biosimilars. Ann Oncol. 2008;19(3):411-9

6. World Health Organization. International Nonproprietary Names [homepage on the Internet]. [cited 2014 Sep 9]. Available from: www.who.int/medicines/ services/inn/en

7. Wyatt JS. Legislative efforts to limit prescription information sharing between patients and healthcare providers represent a serious threat to the health and safety of the American nation. Generics and Biosimilars Initiative Journal (GaBI Journal). 2013;2(4):165. doi:10.5639/gabij.2013.0204.045

8. Alexander EA. The biosimilar name debate: what's at stake for public health Generics and Biosimilars Initiative Journal (GaBI Journal). 2014;3(1):10-2. doi:10.5639/gabij.2014.0301.005

9. Dolinar RO, Reilly MS. Biosimilars naming, label transparency and authority of choice - survey findings among European physicians. Generics and Biosimilars Initiative Journal (GaBI Journal). 2014;3(2):58-62. doi.10.5639/gabij.2014.0302.018

DOI: 10.5639/gabij.2014.0304.045

Copyright (C) 2014 Pro Pharma Communications International 